\title{
Price of defining common time
}

\section{Defining time in relatively moving frames of reference so as to preserve the commonsense notion of simultaneity is possible, but only at an unacceptable price.}

THOSE who from time to time send to Nature, as manuscripts intended for publication, proofs that the special theory of relativity must be wrong will in future, at least in the first instance, be referred to the issue of Il Nuovo Cimento $(74 \mathrm{~B}, 67 ; 1983)$ in which Dr J. P. Hsu from the National Aeronautics and Space Administration's Goddard Space Flight Center works out some of the consequences of rejecting the notion that the velocity of light is the same in all frames of reference moving relative to each other (or, more strictly, in all inertial frames). For although Dr Hsu's professed objective is to demonstrate that some "fundamental" constants are more fundamental than others, he embarks on his argument by throwing away the assumption that the velocity of light is constant (and isotropic) for the sake of a system for measuring time which satisfies one of the rudimentary goals of the anti-relativists doing away with problems of simultaneity. In passing, and inevitably, he shows that the game cannot be worth the candle.

Hsu's starting point, explicitly and emphatically not that of an anti-relativist, is that it should be philosophically permissible to require a system for the measurement of what he calls "common time" that should be valid in all relatively moving frames of reference. The procedure is straightforward, and follows Einstein's original discussion of the problem. Within one frame of reference, a system of identical clocks ticking at what is supposed to be the same rate can easily be synchronized by means of light signals exchanged between observers attending the various instruments. Similarly, a population of clocks in a relatively moving frame of reference can be synchronized among themselves, and then there is nothing to prevent those in charge of the second system from adjusting the rate at which each in the set of moving clocks ticks so that the two systems always tell the same time. And so on, to other systems moving with different relative velocity. So there is no practical obstacle to the construction of a system of common time in which people's commonsense expectations will not be offended. The other consequences, however, will be less palatable.

For the purposes of argument, it is sufficient to consider two linear frames of reference $S$ and $S^{\prime}$, with the second moving relative to the first with velocity $v$. In standard special relativity, the transformation from one system to another is easily ac- complished by the relations $x^{\prime}=y(x-c t)$ and $c^{\prime}=\gamma(c t-\beta x)$ where $x, x^{\prime}$ and $t, t^{\prime}$ are respectively the coordinates and times in the two frames, where $c$ is the velocity of light, $\beta$ is the ratio $v / c$ and $\gamma$ is the familiar $\left(1-\left(v^{2} / c^{2}\right)^{-1 / 2}\right.$. Implicit in these relations is the assumption that the velocity of light is $c$ in both frames of reference. If, however, it is the time that remains unchanged, the standard equation for the transformation of time must be changed into a form that Hsu writes as $b^{\prime} \mathrm{t}=\gamma(c t-\gamma x)$, where $b^{\prime}$ is neither the velocity of light in the second frame nor even, for that matter, a constant. But it does turn out that the velocity of light in the second frame is given by $c^{\prime}=\gamma(c-v)$, from which it is in no sense surprising that, on the assumption that the velocity of light is isotropic (the same in both directions) in the first frame, it turns out to be anisotropic in the second.

Logically, this is a serious stumbling block for anti-relativists who may have chosen to follow Hsu thus far, for if it is accepted that either frame can be chosen as a starting point for a system of universally synchronous clocks, the notion that the velocity of light in all other frames should be anisotropic is much more absurd than the usual anti-relativists' complaint about the twin "paradox". As it happens, Hsu does show that the anisotropy of the velocity of light in moving frames of reference should not affect measurements of the velocity of light as obtained from a roundtrip measurement as in the MichelsonMorley experiment, whose null result is therefore not an absolute bar to attempts to construct a system for the measurement of time in which the difficulties of simultaneity simply melt away.

$\mathrm{Hsu}$, of course, is bent on frying other fish and, in particular, on finding criteria by which the different fundamental constants can be distinguished from each other. For this purpose, he manipulates the laws of motion for a charged particle in an electromagnetic field in such a way that they remain invariant when transformed according to the rules appropriate to common time, not standard special relativity. Hsu chooses to modify the action integral or, which comes to the same thing, the lagrangian which is its integrand, but the job could no doubt be done in other ways.

One obvious consequence, however, stems from the mere form of the equations of motion of, say, an electron in an electromagnetic field, where the particle is coupled to the field by means of the factor $e / c$, where $e$ is the charge of the electron in electrostatic units. So what happens if the velocity of light changes from one frame of reference to another? Plainly it is easiest to formulate equations of motion that remain invariant if the factor by means of which the components of the electromagnetic four-potential are multiplied, $e / c$, remains invariant, and Hsu shows that this is indeed a consequence of his argument. But $e / c$ is nothing but the charge of the electron in electromagnetic units, $\bar{e}$, which is thus singled out as a kind of preferred fundamental constant. By a similarly straightforward consideration of the relationship between the momentum of a particle and the wavenumber of the corresponding matter wave, Hsu also concludes that Planck's constant $\hbar$ (strictly, Planck's constant divided by 2 ), is not strictly a fundamental constant, but that the ratio $\hbar / c$, called $J$, should remain numerically invariant during transformation within the framework of proper time just as in special relativity proper. He points out that the value of the fine structure constant, whose value is $\vec{e}^{2} / J$, also remains a constant and thus retains in a system of common time its value of about $1 / 137$.

The significance of this argument will vary from one reader of it to another. Many will no doubt take the view that since the whole weight of experimental evidence supports standard special relativity, in which the velocity of light is strictly constant from one frame to another moving relative to it, it is a strictly academic exercise to attempt to distinguish between constants supposed on Hsu's argument to be composite and constants such as $h / c$ which are strictly constant both in special relativity and in common time. Hsu's own justification is that, if the equations of motion are modified to allow for universal time, opportunities for using electromagnetic phenomena for telling the difference between one system and another are many fewer than would be expected. Hsu also argues that a system of common time would make intelligible concepts which are now hard to grapple with, such as that of a wave function purporting to describe the probability distribution of a particle at some instant of time.

A better way of tackling this problem may be that suggested by Panafiel and Rafanelli (Il Nuovo Cimento 72B, 157; 1982) who showed how it is possible to treat a classical system of interacting particles without violating Einstein's concept of time. 